\title{
Clues to dwarf galaxy formation from clustering and kinematics
}

\author{
James E. Taylor ${ }^{1}$, Joe Silk ${ }^{2}$ \\ and Arif Babul ${ }^{3}$ \\ ${ }^{1}$ Astronomy Department, California Institute of Technology, \\ mc 105-24, 1200 E. California Blvd., Pasadena, CA 91125, USA \\ email: jet@astro.caltech.edu \\ ${ }^{2}$ Astrophysics, University of Oxford, \\ Denys Wilkinson Building, Keble Road, Oxford, OX1 3RH, UK \\ email: silk@astro.ox.ac.uk \\ ${ }^{3}$ Department of Physics and Astronomy, University of Victoria, \\ Elliott Building, 3800 Finnerty Road, Victoria, B.C., V8P 1A1, Canada \\ email: babul@uvic.ca
}

\begin{abstract}
If the CDM paradigm is correct, then dwarf galaxies are far rarer than dark matter haloes of comparable circular velocity at the present day. Some process or processes must suppress galaxy formation almost completely on these small scales. Several plausible mechanisms may explain the scarcity of dwarf galaxies in the local universe, but it has been hard to distinguish between the different models observationally. The spatial distribution and kinematics of dwarf galaxies contain additional information about the age and formation history of these systems. Interpreted in the CDM framework, they provide powerful new constraints on the physics of dwarf suppression, ruling out at high significance the simplest models, in which the halo occupation probability is constant above some fixed mass or velocity threshold. We describe recent tests of small-scale galaxy formation models using the satellites of the Local Group and other nearby groups, and discuss the implications of these results for studies of the stellar halo, sub-galactic dark matter and the reionization of the universe at high redshift.
\end{abstract}

Keywords. dark matter; galaxies: formation, dwarf, Local Group; Galaxy: halo

\section{Introduction}

In the current cosmological model, cold dark matter (CDM) haloes are the sites of all galaxy formation. Haloes build up progressively through hierarchical merging, such that the most massive haloes at any epoch are also the 'youngest', having assembled their mass into a single structure most recently. A naïve application of this trend to galaxy formation suggests that massive galaxies like the Milky Way should have formed recently, while small galaxies like the dwarf spheroidals of the Local Group may be 'building blocks' left over from an earlier era of structure formation. This picture seems consistent with some observations - the chemical evidence for continuous infall of new gas into the Milky Way, for instance - but fails to explain many others, notably the lack of active star formation in cluster ellipticals, the most massive galaxies in the present-day universe, and its continued presence in field dwarf galaxies.

At least part of this apparent disagreement stems from neglecting the other strong correlation observed in CDM simulations, between age and environment. Dense environments produce structure on any given scale faster than low-density environments, and consequently we expect the oldest galaxy-mass systems to be in rich clusters and the youngest to be in the field and in voids. Applying the same logic to smaller scales, dwarf 
galaxies within virialized systems, such as the dozen small satellites orbiting in the halo of the Milk Way, should be older than similar objects seen in the field, with a continuous gradient in age extending from one environment to the other.

This trend in age is particularly interesting when combined with the other puzzling prediction from CDM models. Simulations of CDM halo formation suggest that the Milky Way should be surrounded by hundreds of dark matter cores dense enough to host dwarf galaxies, whereas in practice only a dozen luminous satellites are observed. While there are several plausible ways of suppressing galaxy formation on these scales - the heating effects of reionization or internal feedback from supernovae, for instance - it has been hard to test and distinguish between these different scenarios observationally.

The age information inherent in the positions and kinematics of the local dwarfs provides a new way of addressing this problem. Comparing the observed distributions to semi-analytic models of halo substructure, we will show evidence that dwarf galaxies populate the oldest subcomponents of the dark matter halo around the Milk Way, rather than the most massive ones. This implies a change in the efficiency of dwarf galaxy formation with redshift, favouring external feedback models over internal ones.

\section{Modelling the Local Satellite Distribution}

We model the evolution of substructure in the dark matter halo of a system like the Milky Way using the semi-analytic model described in Taylor \& Babul (2004). This model combines Press-Schechter merger histories, which predict when subhaloes merge into the halo of the main system, with an analytic description of orbital evolution and mass loss, which determines how they evolve subsequently. While the model is approximate in many ways, it has shown good agreement with self-consistent numerical simulations at the highest resolution (Taylor \& Babul 2005a,b). The model produces catalogues of subhaloes orbiting within the final system, with full information about their current and prior mass, structure and orbit.

Using this model we generated a set of 144 merger trees for a concordance LCDM cosmology $\left(\Lambda=0.7, \Omega_{\mathrm{m}}=0.3, h=0.7, \sigma_{8}=0.9\right)$. Each model halo had a final mass of $1.6 \times 10^{12}$, consistent with mass estimates for the Milky Way, and in each system we also modelled the dynamical effects of a growing galactic component, letting a disk and bulge build up following the prescriptions described in Islam, Taylor \& Silk 2003. In each halo, we then identified the 'first-ranked' subhaloes at the present day, ranking subhaloes by their original peak circular velocity $V_{\text {peak, } 0}$, that is the maximum value this quantity attained at any point in the system's past.

Figure 1 compares the positions and velocities of first-ranked systems $\left(V_{\text {peak }, 0}>\right.$ $45 \mathrm{~km} \mathrm{~s}^{-1}$ ) in a few of our model haloes with the same quantities for the known satellites. The spatial spatial distribution of the first-ranked subhaloes is clearly very different from that of the observed satellites, which lie at smaller galactocentric radii and at negative velocities. Overall, fewer than $1 \%$ of our model haloes have their dozen first-ranked satellites as tightly clustered as the satellites of the Milk Way. Given the age gradient in substructure with galactocentric distance, the simplest way to account for the different distributions is to suppose that dwarf galaxies occupy the oldest subhaloes preferentially.

\section{Implications}

Dark Satellites: Figure 1 shows that the visible satellites are more clustered around the Milky Way than its most massive subhaloes should be. One immediate implication is that some of the largest dark matter structures nearby contain no visible stars. Such 


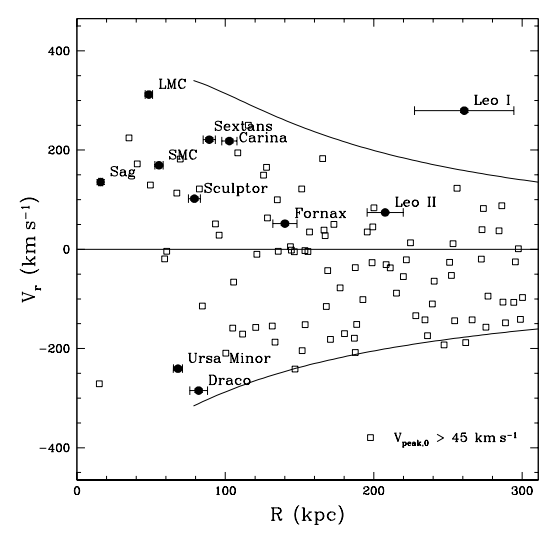

Figure 1. Radial velocity vs. position for the known galactic satellites (solid points with error bars) and massive subhaloes in a set of model haloes (filled squares).

massive $\left(\sim 10^{9}-10^{10} M_{\odot}\right)$ dark satellites might be detectable around distant galaxies that are strong gravitational lenses (Chen, Dalal \& Taylor in preparation).

Reionization: If local dwarf galaxies preferentially occupy the oldest substructures, this implies that dwarf formation was more efficient at high redshift, possibly because of the absence of a strong ionizing background. In fact, dwarf galaxies like those in the Local Group may be the source of much of this ionizing background (Yan \& Windhorst 2004), and enhanced dwarf galaxy formation may explain why the universe was reionized at a relatively high redshift $(z \geqslant 10-15$ ? Spergel et al. 2003) and how it remained ionized during the pre-quasar era $(z \sim 4-6)$.

Tidal Streams: If we assume an efficiency for galaxy formation that decreases with time in such a way as to reproduce the number and spatial distribution of the observed dwarfs, then we can also comment on how many visible satellites may have merged with the Milky Way in the past. We set a threshold in the (original) peak circular velocity $V_{\text {peak,min }}=135 \mathrm{~km} \mathrm{~s}^{-1}(1+z)^{2 / 3}$ above which all haloes are occupied by galaxies, where the normalization is fixed to get the right number of surviving systems and the slope was motivated by the results of Kravtsov Gnedin \& Klypin (2004). Figure 2a shows the properties of the luminous satellites selected using this prescription. We find that if a galaxy like the Milky Way has 10 surviving luminous satellites at the present day, there should be an equal number of heavily stripped or disrupting systems in its halo, together with the debris from ten times as many completely disrupted systems. This suggests that future surveys of the stellar halo could detect abundant substructure. One the other hand, we also find that most of these satellites first merged with the main system more than 10 Gyr ago, so their debris may heavily mixed and hard to isolate in practice.

Satellites in Other Nearby Groups: Our conclusions are based on the properties of the satellites of the Milky Way, which may not be a typical case. It is essential to build up observations of other similar systems to confirm our general conclusions about dwarf galaxy formation. While there are a number of well-studied groups in the local universe (Karachentsev et al. 2005), distances to their satellites are uncertain by hundreds of kpc, leaving only a weaker, projected signature of clustering. Intriguingly, projected distributions of satellites around the Milky Way do appear slightly more clustered than those of other nearby systems (figure 2b). Dwarf galaxies are faint, low surface-brightness objects, however, so catalogues of these objects may be incomplete, especially for objects so close to their parent system they are projected near or on top of it. Further surveys 

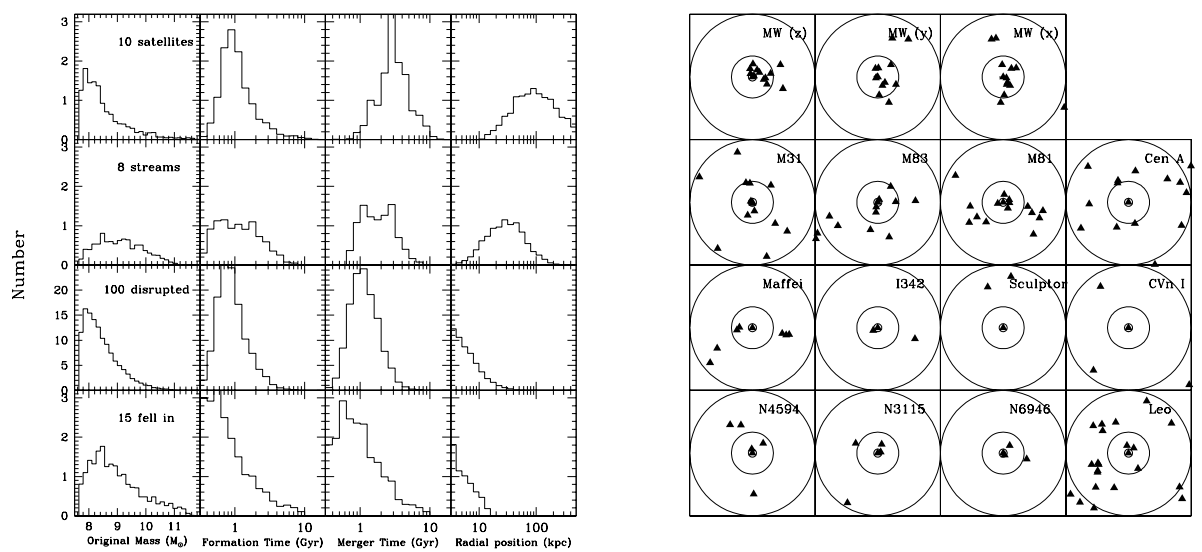

Figure 2. a) Original mass, formation time, merger time, and galactocentric distance (four columns, left to right) for luminous satellites that are intact, heavily stripped, disrupted and centrally merged (four rows, top to bottom) in the model haloes at the present day. b) The dwarf satellites of the Milky Way, projected along three axes (top three panels), compared with the projected distribution of satellites in a number of other local systems (Karachentsev 2005). The small and large circles in each panel indicate 0.3 and 1.0 virial radii respectively, assuming halo mass scales with galaxy mass.

of nearby groups should help fix the strength of dwarf galaxy clustering, giving stronger constraints on dwarf formation models.

\section{Acknowledgements}

The authors gratefully acknowledge helpful discussions with A. Ferguson, A. Kravtsov and I. Karachentsev, as well as financial support from the U.K. Particle Physics and Astronomy Research Council (PPARC), the U.S. National Science Foundation (grant AST-0307859) and the U.S. Department of Energy (contract DE-FG02-04ER41316).

\section{References}

Islam, R.R., Taylor, J.E. \& Silk, J. 2003, MNRAS 340, 647

Karachentsev, I.D. 2005, AJ 129, 178

Kravtsov, A.V., Gnedin, O.Y. \& Klypin, A.A. 2004, ApJ 609, 482

Spergel, D.N. et al. 2003, ApJS 148, 175

Taylor, J.E. \& Babul, A. 2004, MNRAS 348, 811

Taylor, J.E. \& Babul, A. 2005a, MNRAS submitted (astro-ph/0410048)

Taylor, J.E. \& Babul, A. 2005b, MNRAS submitted (astro-ph/0410048)

Yan, H. \& Windhorst, R.A. 2004, ApJ 600, L1

\section{Discussion}

DEKEL: How model-dependent is your argument concerning the clustering of dark halos versus MW dwarfs? How does it compare to N-body simulations?

TAYLOR: The argument is generally model-independent. The only major uncertainty in the modelling concerns highly stripped halos; it is not clear whether these would correspond to dwarfs, tidal streams, or both (e.g., Sagittarius). The N-body simulations find the same result (cf. Diemand's talk). 